Article

\title{
Evaluation of Computational Chemistry Methods for Predicting Redox Potentials of Quinone-Based Cathodes for Li-Ion Batteries
}

\author{
Xuan Zhou ${ }^{1,2,3}$, Abhishek Khetan 1,2,4 (D) and Süleyman Er 1,2,*(D) \\ 1 DIFFER—Dutch Institute for Fundamental Energy Research, De Zaale 20, \\ 5612 AJ Eindhoven, The Netherlands; x.zhou@differ.nl (X.Z.); a.khetan@itv.rwth-aachen.de (A.K.) \\ 2 CCER-Center for Computational Energy Research, De Zaale 20, 5612 AJ Eindhoven, The Netherlands \\ 3 Department of Applied Physics, Eindhoven University of Technology, 5600 MB Eindhoven, The Netherlands \\ 4 Fuel Science Center, RWTH Aachen University, 52062 Aachen, Germany \\ * Correspondence: S.Er@differ.nl
}

Citation: Zhou, X.; Khetan, A.; Er, S. Evaluation of Computational Chemistry Methods for Predicting Redox Potentials of Quinone-Based Cathodes for Li-Ion Batteries. Batteries 2021, 7, 71. https://doi.org/ $10.3390 /$ batteries7040071

Academic Editors: Karim Zaghib, Yuesheng Wang and Seung-Wan Song

Received: 19 July 2021

Accepted: 18 October 2021

Published: 28 October 2021

Publisher's Note: MDPI stays neutral with regard to jurisdictional claims in published maps and institutional affiliations.

Copyright: (c) 2021 by the authors. Licensee MDPI, Basel, Switzerland. This article is an open access article distributed under the terms and conditions of the Creative Commons Attribution (CC BY) license (https:// creativecommons.org/licenses/by/ $4.0 /)$.

\begin{abstract}
High-throughput computational screening (HTCS) is an effective tool to accelerate the discovery of active materials for Li-ion batteries. For the evaluation of organic cathode materials, the effectiveness of HTCS depends on the accuracy of the employed chemical descriptors and their computing cost. This work was focused on evaluating the performance of computational chemistry methods, including semi-empirical quantum mechanics (SEQM), density-functional tightbinding (DFTB), and density functional theory (DFT), for the prediction of the redox potentials of quinone-based cathode materials for Li-ion batteries. In addition, we evaluated the accuracy of three energy-related descriptors: (1) the redox reaction energy, (2) the lowest unoccupied molecular orbital (LUMO) energy of reactant molecules, and (3) the highest occupied molecular orbital (HOMO) energy of lithiated product molecules. Among them, the LUMO energy of the reactant compounds, regardless of the level of theory used for its calculation, showed the best performance as a descriptor for the prediction of experimental redox potentials. This finding contrasts with our earlier results on the calculation of quinone redox potentials in aqueous media for redox flow batteries, for which the redox reaction energy was the best descriptor. Furthermore, the combination of geometry optimization using low-level methods (e.g., SEQM or DFTB) followed by energy calculation with DFT yielded accuracy as good as the full optimization of geometry using the DFT calculations. Thus, the proposed calculation scheme is useful for both the optimum use of computational resources and the systematic generation of robust calculation data on quinone-based cathode compounds for the training of data-driven material discovery models.
\end{abstract}

Keywords: computational chemistry; semi-empirical calculations; DFT calculations; quinones; Li-ion batteries; energy storage

\section{Introduction}

Lithium-ion batteries (LIBs) are some the most widely investigated energy systems designed to meet the ever-increasing demands for renewable energy storage and thereby to alleviate global environmental issues [1]. Characterized by high-abundance, low cost, and relatively sustainable synthesis and recycling procedures, organic electroactive materials have received increasing attention as high-performance battery electrodes [2,3]. They are also structurally diverse, which allows one to tune their redox and mechanical properties [3]. Among the currently investigated classes of organic materials, conjugated carbonyls are widely studied because of their tunable structures and battery-relevant properties [4].

Quinones, which are a broad subclass of conjugated carbonyls, have drawn huge interest from the research community, especially because of their reversible electrochemistry with $\mathrm{Li}$ ions [5]. The redox mechanism of quinones can be explained by the enolate 
reactions on the carbonyl groups [6] that are stabilized by conjugation. However, quinonebased compounds suffer from high solubility in organic electrolytes, and their redox properties require further optimization for practical application as cathode materials $[4,7]$. Therefore, various modifications, such as functionalization with chemical groups [8], the substitution of heteroatoms [9], the fusion of aromatic rings [10], and increasing the number of carbonyl groups [11], have been used to tune their intrinsic properties to improve their electrochemical performance. Given the large chemical and configurational space of electroactive compounds, high-throughput computational screening (HTCS) is a promising strategy for creating virtual libraries of diverse electroactive compounds, predicting their performance by computing descriptors, and identifying the most promising candidates for further validation [12-17].

Typically, HTCS studies use quantum chemical simulations for the prediction of redox properties; hence, the computational cost associated with screening possibly millions of candidate compounds can become unfeasibly large. Therefore, the performance descriptors used in HTCS need to be carefully chosen, and the trade-offs between their accuracy and computing cost must be addressed. One of the central properties of interest is the redox potential of the lithiation of carbonyl groups because it is directly proportional to the cell voltage and energy density. Multiple studies have reported the reasonably accurate $(\sim 50 \mathrm{mV})$ prediction of the redox potential of quinones for redox flow battery applications, in which they are treated in a single-molecule (gas phase) model with implicit solvation $[13,18]$. However, the computational prediction of redox potentials with the explicit treatment of the amorphous or crystalline electrodes is a difficult challenge because it requires knowledge of the solid-state structure [19].

Although it is in principle possible to achieve higher prediction accuracy with $a b$ initio simulations of the solid phase [20], such an approach is in practice computationally demanding because the amorphous or semi-amorphous nature of these solids requires the large simulation cells and an exhaustive search for the lowest-energy structure over a typically intractable configurational space. Given the fact that the subsequent lithiation of these structures will arguably increase the complexity multifold because many equally probable amorphous structures of even the same compound have varying charge capacities and redox potentials, HTCS using ab initio methods is currently infeasible for the solid-phase structures of thousands of quinone-based candidates. Therefore, a common approach for estimating the redox potentials of quinones is to perform simulations of gas phase molecules using density functional theory (DFT) $[8,11,21]$. Although DFT has been extensively applied for performing such computations, other less frequently used low level methods, such as semi-empirical quantum mechanics (SEQM) and density functional tight binding (DFTB), hold promise to speed up structure and property predictions while retaining accuracy.

In the current work, we systematically evaluated the performance of the level of theories, including DFT, DFTB, SEQM, and their hybrid combinations, for predicting the redox potentials of quinone-based compounds within the gas phase model. The effectiveness of this approximation was validated in the context of different structural variations of the molecular backbones, including heteroatom substitutions and functionalization with chemical groups. For the prediction of experimental redox potentials, we compared the performance of three descriptors by independently calibrating them against the available experimental electrochemical data of the compounds. These descriptors included the reaction energy of molecules with $\mathrm{Li}$ atoms, the lowest unoccupied molecular orbital (LUMO) energy of the reactant, and the highest occupied molecular orbital (HOMO) energy of the lithiated product molecules. The performance of descriptors and calculation procedures for the acceleration of redox potential prediction of vast chemical libraries were analyzed. 


\section{Methods}

\subsection{Choice of Descriptors}

Within the gas phase approximation, the redox potential $\left(E^{\mathrm{o}}\right)$ can be described using the Nernst equation as follows:

$$
E^{\mathrm{o}}=-\frac{\Delta G_{r}}{n F}
$$

where $\Delta G_{r}$ denotes the Gibbs free energy difference between the reactant and product, $F$ is the Faraday constant, and $n$ is the number of transferred electrons. $\Delta G_{r}$ can be expressed as:

$$
\Delta G_{r}=\Delta E_{r}+p \Delta V_{r}-T \Delta S_{r}+\Delta(Z P E)
$$

where $\Delta E_{r}$ is the change in internal energy, also called reaction energy. $\Delta V_{r}, \Delta S_{r}$, and $\triangle(Z P E)$ correspond to changes in volume, entropy, and zero-point energy, respectively. Pressure and temperature are represented by $p$ and $T$, respectively. In the gas phase approximation, the terms $p \Delta V_{r}$ and $T \Delta \mathrm{S}_{\mathrm{r}}$ are typically much smaller than $\Delta E_{r}$ [19], and they are therefore neglected in the equation. For the prediction of the redox potentials of quinone-based organic molecules, $\triangle(Z P E)$ is sufficiently small (as shown in Table S10) and can be neglected in HTCS studies $[13,22]$. Thus, the redox potential is approximated as:

$$
E^{o}=-\frac{\Delta E_{r}}{n F}
$$

where $\Delta E_{r}$ is given by:

$$
\Delta E_{r}=E_{Q L i_{2}}-E_{Q}-2 E_{L i}
$$

where $E_{Q L i_{2}}, E_{Q}$, and $E_{L i}$ denote the total energy of a lithiated molecule, reactant quinone molecule, and a lithium atom in gas phase, respectively. In addition to $\Delta E_{r}$, we also considered the frontier orbital energies corresponding to the HOMO of the lithiated molecule and the LUMO of the reactant molecule as descriptors for the prediction of redox potentials [23].

The prediction performance of redox potential can be evaluated by calculating the coefficient of determination $\left(\mathrm{R}^{2}\right)$ and root-mean-square error (RMSE) of the linear regression (LR) between the computational data and the measured redox potentials. To facilitate the comparison between models with different scales, we used the normalized RMSE (NRMSE) [24], which is defined as the RMSE for any given dataset divided by the range of redox potentials spanned by that dataset:

$$
\text { NRMSE }=\frac{\text { RMSE }}{\text { Range of experimental redox potential }}
$$

\subsection{Experimental Data for Validation}

In order to examine the validity of the gas phase approximation, we collected experimental data of the lithiation redox potential of a wide variety of quinone-like electroactive compounds for Li-ion batteries [8-11,21,25-28]. We separately performed LR analysis on the groups of molecules from different literature sources, thus ensuring consistency with respect to electrode synthesis, electrolyte formulation, cell configurations, charge/discharge rates, and other experimental conditions. While numerous experimental studies have been reported on quinone-like electrodes, we identified seven experimental datasets [8-11,21,26,27], as shown in Table 1, that satisfied the following five criteria: (1) the total number of different compounds that have been tested should be at least three, which is essentially the minimal number of points for a LR model; (2) the redox potentials of the different molecules should span a range that is wider than $0.1 \mathrm{~V} \mathrm{vs.} \mathrm{Li} / \mathrm{Li}^{+}$, as this value is similar to errors in DFT calculations [29]; (3) the redox potentials of compounds that involve a two-electron lithiation process should be clearly specified rather than be presented as a range of values [28]; (4) the lithiation sites on the molecules should be limited to the carbonyl groups that are directly attached to the rings of quinones, as opposed to the chemical functional groups of the molecules; and (5) molecules with more than four 
rings should be ignored because it is known that the $\pi-\pi$ stacking interaction between quinones rapidly increases with the number of aromatic rings [30] and the intermolecular interactions consequently increase, which is likely to result in errors within the gas phase approximation [20]. In addition to these criteria, fluorinated 2, 2'-bis- $p$-benzoquinone $\left(\mathrm{F}_{2}-\right.$ BBQ) [21] from dataset No. 5 was removed because of its fast decomposition (12\% capacity retained after 20th cycle) and therefore lack of discharge voltage data. As a result, a total of 39 compounds divided into seven sets were identified for the validation of the descriptors and computational models. The two-dimensional (2D) structures of these compounds are shown in Figure 1.

Table 1. A summary of experimental data for each of the seven calibration datasets.

\begin{tabular}{|c|c|c|c|c|c|}
\hline Dataset & Electrolyte & $\begin{array}{l}\text { Number of } \\
\text { Molecules }\end{array}$ & $\begin{array}{l}\text { Discharge } \\
\text { Condition }\end{array}$ & $\begin{array}{l}\text { Range of Redox } \\
\text { Potential (vs. } \\
\text { Li/Li+ }\end{array}$ & Data Source * \\
\hline 1 & $1 \mathrm{M} \mathrm{LiPF}_{6}-\mathrm{EC}+\mathrm{DEC}(v / v=3: 7)$ & 4 & $0.1 \mathrm{~mA}$ & $0.60 \mathrm{~V}$ & Table \\
\hline 2 & $1 \mathrm{MLiPF}_{6}-\mathrm{EC}+\mathrm{DMC}(w / w=1: 1)$ & 4 & $1 \mathrm{Li}$ per $5 \mathrm{~h}$ & $0.44 \mathrm{~V}$ & Text \\
\hline 3 & $1 \mathrm{MLiPF}_{6}-\mathrm{EC}+\mathrm{DMC}(w / w=1: 1)$ & 5 & $1 \mathrm{Li}$ per $5 \mathrm{~h}$ & $0.66 \mathrm{~V}$ & Table \\
\hline 4 & $1 \mathrm{M} \mathrm{LiPF}_{6}-\mathrm{EC}+\mathrm{DMC}(v / v=3: 7)$ & 6 & $1 \mathrm{mV} / \mathrm{s}$ & $1.55 \mathrm{~V}$ & Text \\
\hline 5 & 2.75 M LiTFSI-Tetraglyme & 8 & $40 \mathrm{~mA} / \mathrm{g}$ & $0.30 \mathrm{~V}$ & Table \\
\hline 6 & 1 M LiTFSI-Tetraglyme & 5 & $40 \mathrm{~mA} / \mathrm{g}$ & $1.00 \mathrm{~V}$ & Table \\
\hline 7 & $1 \mathrm{M} \mathrm{LiPF}_{6}-\mathrm{PC}$ & 7 & $1 \mathrm{Li}$ per $10 \mathrm{~h}$ & $0.82 \mathrm{~V}$ & Text \\
\hline
\end{tabular}

* Table and Text indicate the original source in the corresponding literature from which the data were extracted.

1

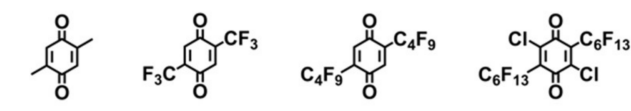

2<smiles>C1=CC23OC2(O1)O3</smiles>

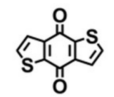

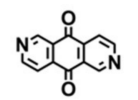

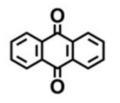

3
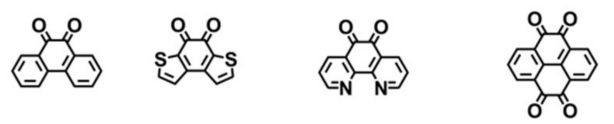<smiles>C1=[Ge]C[Ge]1</smiles>

4<smiles></smiles>

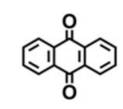

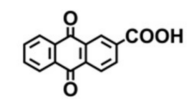

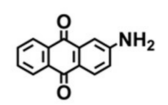

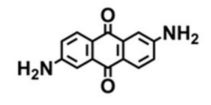

5<smiles>c1coc2=c3ccoc(co3)-c-2c1</smiles>

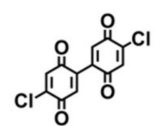

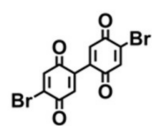

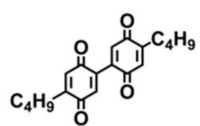

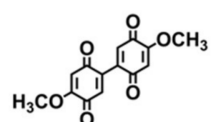

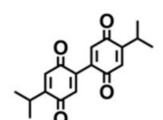

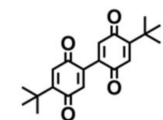

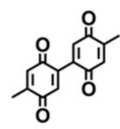

6<smiles>C1=Cc2cc1co2</smiles><smiles>C1=CC23COC2(C=C1)O3</smiles>
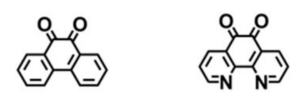<smiles>C1O[Ge]1</smiles>

7

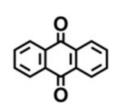
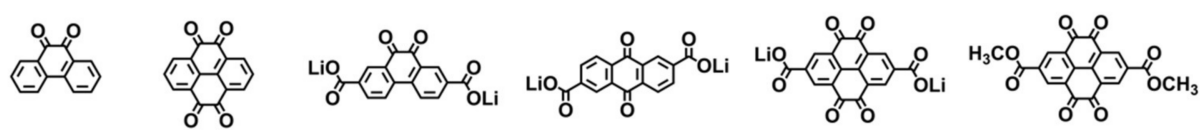

Figure 1. 2D structures of the molecules that were used for the calibration of computational methods. A total of seven different experimental datasets were considered in the current study.

As shown in Figure 1, the experimentally tested compounds exhibit good structural diversity in terms of quinone-based backbones and their functional derivatives. The molecules contain a maximum of four rings and four $\mathrm{C}=\mathrm{O}$ groups as lithiation sites, which are located at the para and ortho positions on the rings. Apart from the heteroatomic substitutions of ring carbons with $\mathrm{N}, \mathrm{O}$, and $\mathrm{S}$ atoms, several compounds are functionalized with a wide selection of electron-donating and -withdrawing groups, including $-\mathrm{CH}_{3},-\mathrm{CF}_{3}$, $-\mathrm{C}_{4} \mathrm{~F}_{9},-\mathrm{C}_{6} \mathrm{~F}_{13},-\mathrm{Cl},-\mathrm{COOH},-\mathrm{NH}_{2},-\mathrm{CH}_{3},-\mathrm{Br},-\mathrm{C}_{4} \mathrm{H}_{9},-\mathrm{CH}\left(\mathrm{CH}_{3}\right)_{2},-\mathrm{C}\left(\mathrm{CH}_{3}\right)_{3},-\mathrm{OCH}_{3}$, $-\mathrm{COOLi}$, and $-\mathrm{COOCH}_{3}$. The measured values of redox potentials were obtained by two 
different experimental techniques: galvanostatic cycling and cyclic voltammetry. Further details on the electrolytes, discharge rates, and type of data source that was used to obtain the measured redox potentials are summarized in Table 1, and the operation voltage and charge/discharge windows for the seven datasets are tabulated in Table S9.

\subsection{Computational Scheme}

To compare the accuracy of various theoretical methods for the prediction of redox potentials, we propose a computational workflow that includes the following steps:

(1) The three-dimensional (3D) molecular geometries were initially created by using the Maestro editor in the Schrödinger Materials Science Suite (version 2019-3) [31].

(2) A search for the lowest energy conformer was performed for all the compounds using the OPLS3e [32] force field.

(3) The lowest energy conformers were further optimized in the gas phase with various SEQM, DFTB, and DFT methods that are described below. As an additional step, single point energy (SPE) calculations using two representative DFT methods were performed on frozen atom coordinates obtained from the SEQM or DFTB optimizations. Altogether, these optimizations yielded descriptor data that were obtained at three levels of approximation: SEQM or DFTB, DFT, and a hybrid of the two.

(4) To explore the possible contributions of solvation effects (as explored in previous studies $[18,33])$, SPE calculations were performed again in an implicit solvation environment within the standard Poisson-Boltzmann Formalism (PBF) [34], in which the parameters for the solvent phase were set according to the experimental conditions from each dataset.

The conformational search was performed by using the MacroModel [35] module. The MOPAC [36] and Jaguar [37] programs, both as implemented in Schrödinger Materials Science Suite, were used to perform the SEQM and DFT calculations, respectively. The DFTB calculations were carried out using the ADF software [38]. Seven SEQM methods were considered here to perform the geometry optimizations: AM1, MNDO, MNDOD, PM3, PM6, PM6-D3H4X, and PM7. GFN1-xTB [39] and SCC-DFTB [40] with the parameter set of QUASINANO2015 [41] were selected for the DFTB based geometry optimizations. Two exchange-correlation functionals, also with and without Grimme's D3 dispersion corrections, were employed in the DFT simulations; these included PBE, PBE-D3, B3LYP, and B3LYP-D3. The LACVP ${ }^{++* *}$ [42] basis set with polarization and diffuse functions was used for the DFT calculations. For DFT optimization calculations, we used grids with medium point density, whereas for DFT SPE calculations, we used finer grids. The hybrid scheme, i.e., the DFT calculated SPE on the SEQM- or DFTB-optimized coordinates, was performed without the dispersion corrections, as they showed no significant advantage when using the full DFT-based calculation scheme. The dielectric constant, molecular weight, and density of the electrolyte solvent used for the SPE calculations involving the implicit solvation effect are provided in Supporting Information Table S1. The values for binary solvents were calculated based on their molar ratios in the mixtures.

\section{Results and Discussions}

To identify an optimal descriptor and prediction method for HTCS, we developed LR models between the experimental redox potentials and the calculated descriptors $\left(\Delta E_{r}\right.$, LUMO, and HOMO energies) for each of the seven datasets. The scatter plots for the NRMSE of the three descriptors that were calculated at three rungs of a computational ladder (DFT, SEQM, and DFTB) are shown in Figure 2, and their numerical data are provided in Tables S2-S4. The corresponding $\mathrm{R}^{2}$ and RMSE data are shown in Supporting Information Figure S1, Tables S5-S7 and S11-S13. We begin with a discussion of the performance of the three descriptors at the highest level of theory considered in this work; the DFT calculated results as shown in Figure 2a-c. Firstly, when using HOMO energy as the descriptor, there was considerable spread in NRMSE values for the various DFT methods for all datasets (Figure 2c). Secondly, when using $\Delta E_{r}$ (Figure 2a), the NRMSE 
spread was $\sim 10 \%$, except for dataset No. 2 , for which the NRMSE ranged from $3.82 \%$ to $29.78 \%$. Thirdly, when the reactant molecule's LUMO energy was used as the descriptor (Figure 2b), all DFT methods showed quite similar performance for each dataset, with an NRMSE spread of approximately 5\%. A consensus between the various DFT methods applied in the current study reveals problems with the optimized structures and the corresponding energies of the lithiated molecules. In addition, when considering DFT methods without implicit solvation, LUMO energy was found to be the best descriptor for predicting the redox potentials not only because of the high consistency between various DFT methods on NRMSE but also because of its lowest prediction error among three descriptors.
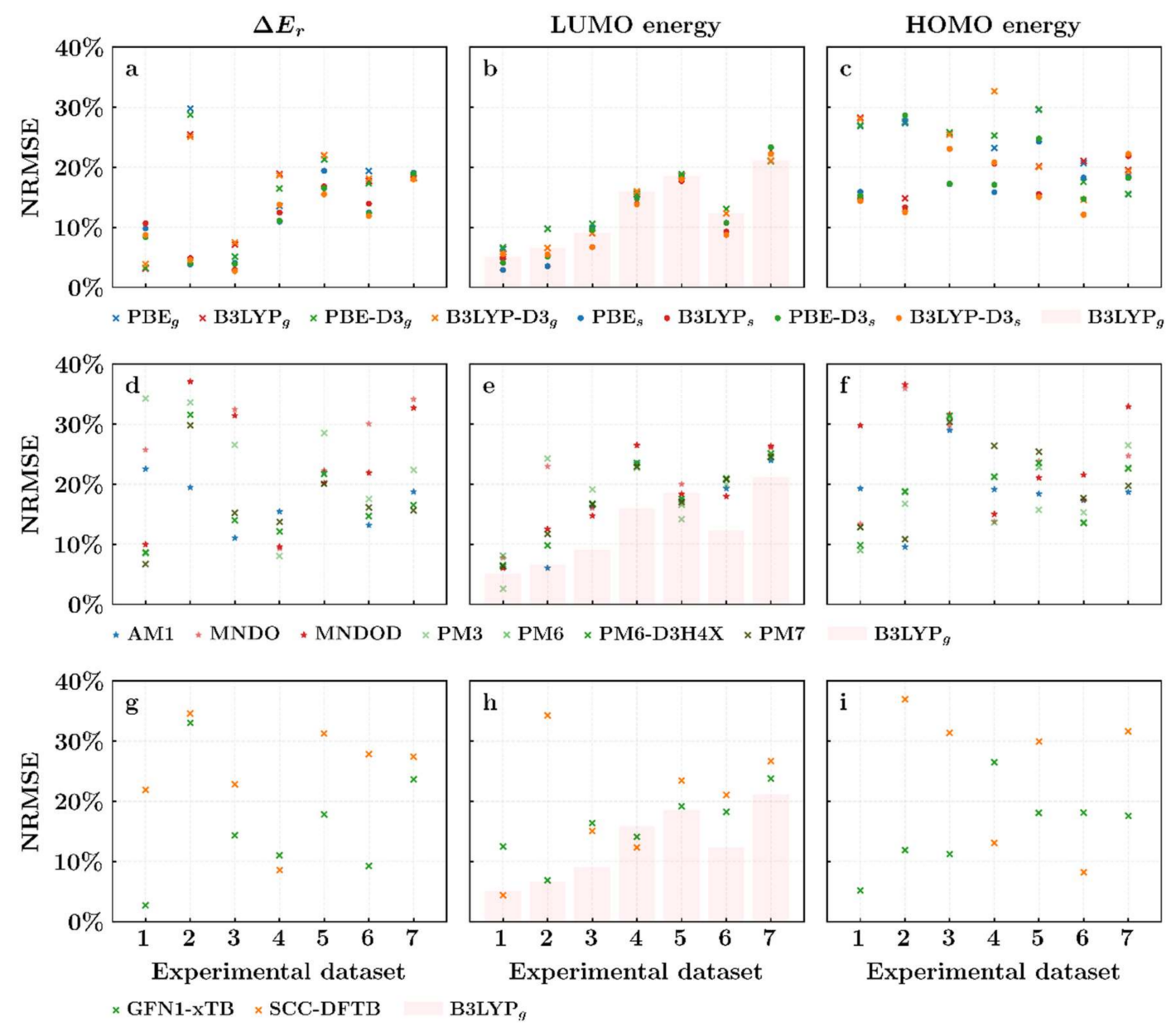

Figure 2. NRMSE of three descriptors- $\Delta E_{r}$, LUMO energy, and HOMO energy-calculated with (a-c) DFT functionals, (d-f) SEQM methods, and (g-i) DFTB methods for the seven datasets. $\mathrm{PBE}_{g}$ and $\mathrm{PBE}_{s}$ represent the calculation of single point energy in the gas $(g)$ and solvent phases $(s)$, respectively. The shaded vertical bars in $(\mathbf{b}, \mathbf{e}, \mathbf{h})$ show the fully B3LYP $g$ calculated data.

In a recent study, we showed that the redox potential of the concerted proton-electron reduction of quinones in aqueous flow batteries is most efficiently predicted by using $\Delta E_{r}$ as the descriptor [13]. As shown in Figure 2, the relatively better performance of the LUMO energy when used as a descriptor for the redox potential predictions of $\mathrm{Li}$-ion batteries can be explained by noting that the gas phase approximation to the solid-state structures became more erroneous. This was likely because the lithiation led to significant changes in both the solid structures and their associated energies of inter-molecular interactions. Moreover, the computation of lithiated molecules not only led to a significant drop in the prediction accuracy of redox potentials but also doubled the calculation efforts. Therefore, these findings are expected to have major implications for strategizing future HTCS efforts from the standpoint of prediction accuracy and computational cost. The inclusion of 
implicit solvation in the DFT calculations of SPE had varying degrees of effect on prediction performance. The inclusion of solvation improved the energetics of lithiated quinones (though not consistently among the datasets), as can be seen in Figure 2c with a decrease in NRMSE values when using the HOMO energy descriptor. On the other hand, the inclusion of solvation had negligible effect for reactant quinones, as can be seen in Figure $2 b$ with the performance of the LUMO energy descriptor. Since the DFT treatment of lithiated molecules in the gas phase model is prone to errors when predicting the measured potentials (as discussed above) and the fact that the inclusion of implicit solvation requires additional computing power, there is no clear advantage of including implicit solvation models for the calculation of chemical descriptors from the perspective of HTCS.

Regarding the performance of the DFT functionals, when LUMO energy was used as the descriptor, B3LYP performed better than PBE in five datasets and was comparable to PBE in the remaining two datasets (Figure 2b; see also Supporting Information Table S3). Moreover, the dispersion corrections to either of the two DFT functionals had insignificant effects on the prediction performance. Accordingly, we chose the results from the B3LYP functional as our benchmark and compared the results from the low-level theoretical methods to them. When comparing the accuracy of the three descriptors calculated with various SEQM (Figure $2 \mathrm{~d}-\mathrm{f}$ ) and DFTB methods (Figure $2 \mathrm{~g}-\mathrm{i}$ ), the trends in the results showed similarities to those of the DFT simulations. As one can expect, the overall errors for SEQM and DFTB were higher than DFT. Though LUMO emerged as the best performing descriptor again, $\Delta E_{r}$ did not show good performance. In addition, as evidenced by the performance of $\mathrm{HOMO}$ energy as the descriptor, the treatment of lithiated compounds with SEQM and DFTB was significantly more erroneous than with DFT. It must also be noted that even when using the LUMO energy as the descriptor, the SEQM methods had a larger NRMSE spread than the DFT methods. As shown in Table S8, the ranking of SEQM methods based on average of the NRMSE for the seven datasets was: AM1 > PM7 > PM6-D3H4X > PM6 > MNDOD > PM3 > MNDO. In the group of DFTB methods, GFN1-xTB showed a better prediction accuracy than SCC-DFTB for all seven datasets. Based on these results, AM1 and GFN1-xTB emerged as the best performing SEQM and DFTB methods, respectively.

A common feature of DFT, SEQM, and DFTB calculations was that their performance on datasets No. 4, 5, and 7 was comparably worse than on the other sets. In dataset No. 4, the number of rings in molecules varied from one to three, which indicates that the varying degree of $\pi-\pi$ interactions are likely to increase the non-systematic errors when using the gas phase model. In experiments with dataset $\mathrm{No} .5$, the $\mathrm{Cl}$ - and $\mathrm{Br}$-substituted quinones were shown to display high solubility in the electrolyte, and the discharge potentials were obtained by averaging the area under the charge-discharge curves. It is possible that these factors incurred large uncertainties in dataset No. 5. Finally, for HTCS purposes, we note that the prediction accuracies of the SEQM methods were not significantly worse than the DFT benchmark for the LUMO energy descriptor. Although the average NRMSE values of the seven datasets for AM1, PM7, and B3LYP were 16.10, 17.13, and $12.65 \%$, respectively (Table S8), the low-level methods were approximately $10^{3}$ times faster to compute. As shown in Figure 2e, the prediction accuracies of the SEQM methods were almost as good as the DFT simulations for datasets No. 1, 2, and 5, but they were slightly worse for datasets No. 3, 4, 6, and 7. The average NRMSE values for GFN1-xTB and SCC-DFTB were 15.85 and $19.60 \%$, respectively. The DFTB methods showed similar prediction accuracies to DFT for datasets No. 1, 2, 4, and 5, though they were comparably worse for datasets No. 3 and 6.

The hybrid scheme of using DFT calculations of SPE on frozen atom coordinates from SEQM or DFTB optimizations was further explored with the aim to improve the prediction accuracy of low-level methods. The NRMSE, $\mathrm{R}^{2}$, and RMSE of hybrid calculations for three descriptors are shown in Figure 3 and Supporting Information Figure S2, Tables S2-S7 and S11-S13. Two SEQM (AM1 and PM7) and two DFTB (GFN1-xTB and SCCDFTB) methods were used for the geometry optimizations, and these were followed by 
SPE calculations using the B3LYP functional. Yet again, the relative behavior of the three descriptors remained unchanged across all seven datasets, and LUMO clearly emerged as the most accurate descriptor with the lowest NRMSE. Remarkably, when using LUMO as the descriptor, the performance of the hybrid scheme was just as good as the DFT calculations across the seven datasets (Figure $3 b, e$ ). Within the gas phase approximation, the notable similarities between the hybrid scheme and DFT results suggest that the differences between the bare low-level calculations and the full DFT calculations originated from the prediction of energies rather than the difference in geometries. It can be observed that inclusion of implicit solvation led to a miniscule effect on the prediction accuracy when using the LUMO descriptor, which was the same as the case of the full DFT calculations. The average NRMSE of the hybrid scheme PM7 / B3LYP ${ }_{g}(11.46 \%)$ was slightly less than that of AM1/B3LYP ${ }_{g}(13.83 \%)$, which was different than the performance of the bare SEQM calculations. A similar conclusion was reached for the hybrid scheme of DFTB and DFT, for which the SCC-DFTB/B3LYP $(12.01 \%)$ slightly outperformed GFN1-xTB/B3LYP $g$ $(13.11 \%)$, and the bare GFN1-xTB results had smaller average NRMSE values than the bare SCC-DFTB results. According to these findings, the PM7 and SCC-DFTB methods are efficient for geometry optimization, and the AM1 and GFN1-xTB methods are better at predicting energies. In summary, when LUMO energy is used as the descriptor for the prediction of measured redox potentials, the hybrid scheme PM7/B3LYP ${ }_{g}$ is the best choice.
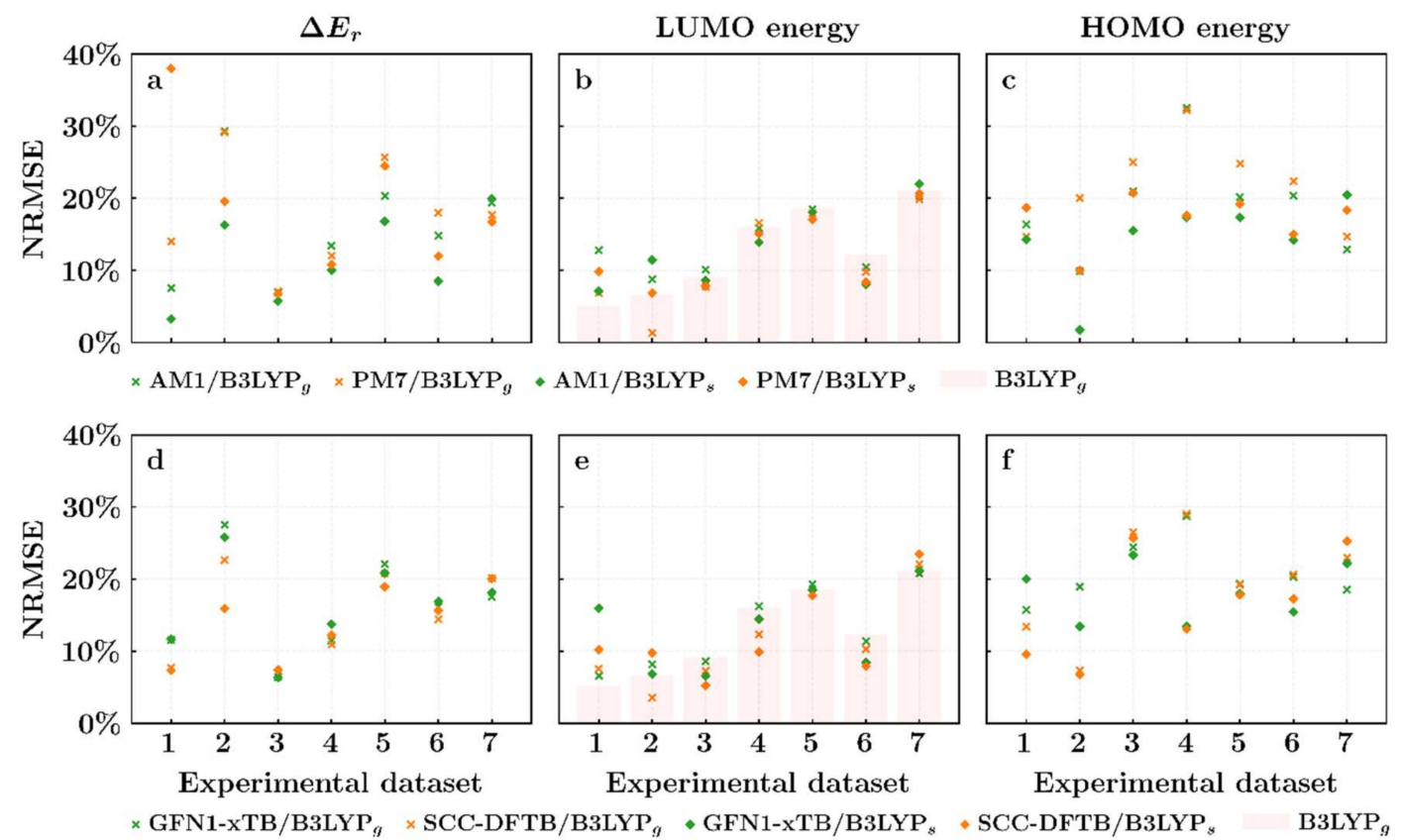

Figure 3. NRMSE of three descriptors: $\Delta E_{r}$, LUMO energy, and HOMO energy. The hybrid schemes of SEQM/DFT $(\mathbf{a}-\mathbf{c})$ and DFTB/DFT (d-f) were used for the seven datasets. Accordingly, AM1/B3LYP $g$ and AM1/B3LYP suggest that the molecules were optimized only with AM1, and their energies were calculated by using B3LYP functional either without or with solvation effect, respectively. The shaded vertical bars in (b,e) show the fully B3LYP $\mathrm{P}_{g}$ calculated data.

\section{Conclusions}

A systematic evaluation of theoretical methods of varying levels of accuracy and computational cost was performed in order to devise an effective strategy for accelerating the prediction of the redox potentials of quinone-based cathode materials for $\mathrm{Li}$-ion batteries. Two DFT functionals with dispersion corrections, seven SEQM and two DFTB parametrizations were considered for evaluation. In addition, hybrid schemes that involve SEQM, DFTB, and DFT methods, were also considered. Three energy-related descriptors $-\Delta E_{r}$, the LUMO energy of the quinones, and the HOMO energy of lithiated quinones-were used against the measured redox potential data of 39 quinone-based cathode materials. 
The LUMO energy of the reactant molecules clearly emerged as the best descriptor at all levels of the considered theory. The inclusion of an implicit solvation model during energy calculations showed a negligible improvement when predicting the redox potentials. Combinations of low-level geometry optimization, either with SEQM or DFTB, and high-level self-consistent single point energy calculation with DFT (B3LYP) offered similar accuracies as the full DFT optimization calculations but at only a fraction of a computational effort. Thus, we anticipate that the evaluation of the performance of descriptors and computational methods, as well as the proposed computational scheme of low- and high-level methods, will be useful for future explorations of the vast chemical space of quinone-based cathode materials for Li-ion batteries.

Supplementary Materials: The following are available online at https:/ /www.mdpi.com/article/10 $.3390 /$ batteries7040071/s1. Table S1: Physical properties, including dielectric constant, molecular weight, and density, of organic electrolytes and their mixtures corresponding to the experimental datasets; Figure S1: $\mathrm{R}^{2}$ of three descriptors: $\Delta E_{r}$, LUMO energy, and HOMO energy. The computational data were calculated by using $(\mathrm{a}-\mathrm{c}) \mathrm{DFT},(\mathrm{d}-\mathrm{f}) \mathrm{SEQM}$, and $(\mathrm{g}-\mathrm{i})$ DFTB methods for the seven experimental datasets. $\mathrm{PBE}_{g}$ and $\mathrm{PBE}_{S}$ represent single point calculations in gas $(g)$ and solvent (s) phases, respectively. The shaded vertical bars in $(b, e, h)$ show the fully $\mathrm{B}^{\mathrm{LYY}} \mathrm{P}_{g}$ calculated data; Figure S2: $\mathrm{R}^{2}$ of three descriptors: $\Delta E_{r}$, LUMO energy, and HOMO energy. The computational data were calculated by using $(\mathrm{a}-\mathrm{c})$ hybrid SEQM/DFT scheme and $(\mathrm{d}, \mathrm{e})$ hybrid DFTB/DFT scheme for the seven experimental datasets. Accordingly, AM1/B3LYP and AM1/B3LYP suggest that the molecules were optimized only with AM1 and their energies were calculated by using B3LYP functional either without or with solvation effect, respectively. The shaded vertical bars in $(b, e, h)$ show the fully B3LYP $\mathrm{P}_{g}$ calculated data; Table S2: NRMSE of $\Delta E_{r}$ as computed with DFT, SEQM, DFTB, and hybrid schemes of SEQM (or DFTB) and DFT methods for the seven datasets; Table S3: NRMSE of LUMO energy of the reactant molecules as computed with DFT, SEQM, DFTB, and hybrid schemes of SEQM (or DFTB) and DFT methods for the seven datasets; Table S4: NRMSE of HOMO energy of the product molecules as computed with DFT, SEQM, DFTB, and hybrid schemes of SEQM (or DFTB) and DFT methods for the seven datasets; Table S5: $\mathrm{R}^{2}$ of $\Delta E_{r}$ as computed with DFT, SEQM, DFTB, and hybrid schemes of SEQM (or DFTB) and DFT methods for the seven datasets; Table S6: $\mathrm{R}^{2}$ of LUMO energy of the reactant molecules as computed with DFT, SEQM, DFTB, and hybrid schemes of SEQM (or DFTB) and DFT methods for the seven datasets; Table S7: $\mathrm{R}^{2}$ of HOMO energy of the product molecules as computed with DFT, SEQM, DFTB, and hybrid schemes of SEQM (or DFTB) and DFT methods for the seven datasets; Table S8: Average NRMSE of the seven datasets for LUMO descriptor as calculated with B3LYP, SEQM, DFTB, and the hybrid schemes of SEQM (or DFTB) and B3LYP; Table S9: Summary of operation voltage and charge/discharge windows for the seven datasets; Table S10: The 2D structures of seven quinones, the zero-point energy (ZPE) of quinone reactants and lithiated products, and their difference in $Z P E$ with the functional of PBE and B3LYP in gas phase; Table S11: RMSE of $\Delta E_{r}$ as computed with DFT, SEQM, DFTB, and hybrid schemes of SEQM (or DFTB) and DFT methods for the seven datasets; Table S12: RMSE of LUMO energy of the reactant molecules as computed with DFT, SEQM, DFTB, and hybrid schemes of SEQM (or DFTB) and DFT methods for the seven datasets; Table S13: RMSE of HOMO energy of the product molecules as computed with DFT, SEQM, DFTB, and hybrid schemes of SEQM (or DFTB) and DFT methods for the seven datasets.

Author Contributions: Conceptualization, S.E.; methodology, X.Z., A.K. and S.E.; modeling and validation, X.Z.; formal analysis and discussion, X.Z., A.K. and S.E.; writing-original draft preparation, X.Z.; writing—review and editing, A.K., S.E. and X.Z.; supervision, A.K. and S.E.; project administration, S.E.; funding acquisition, S.E. All authors have read and agreed to the published version of the manuscript.

Funding: S.E. acknowledges funding from the initiative "Computational Sciences for Energy Research" of Shell and NWO grant No. 15CSTT05. X.Z. acknowledges financial support from the China Scholarship Council grant No. CSC201806240322. This work was sponsored by NWO Exact and Natural Sciences for the use of supercomputer facilities.

Institutional Review Board Statement: Not applicable.

Informed Consent Statement: Not applicable. 
Data Availability Statement: The generated computational data presented in this study are available on request from the corresponding author.

Conflicts of Interest: The authors declare no conflict of interest.

\section{References}

1. Thackeray, M.M.; Wolverton, C.; Isaacs, E.D. Electrical energy storage for transportation-approaching the limits of, and going beyond, lithium-ion batteries. Energy Environ. Sci. 2012, 5, 7854-7863. [CrossRef]

2. Lee, S.; Kwon, G.; Ku, K.; Yoon, K.; Jung, S.K.; Lim, H.D.; Kang, K. Recent progress in organic electrodes for Li and Na rechargeable batteries. Adv. Mater. 2018, 30, 1704682. [CrossRef] [PubMed]

3. Xu, D.Y.; Liang, M.X.; Qi, S.; Sun, W.W.; Lv, L.P.; Du, F.H.; Wang, B.F.; Chen, S.Q.; Wang, Y.; Yu, Y. The progress and prospect of tunable organic molecules for organic lithium-ion batteries. ACS Nano 2021, 15, 47-80. [CrossRef] [PubMed]

4. Zhu, L.M.; Ding, G.C.; Xie, L.L.; Cao, X.Y.; Liu, J.P.; Lei, X.F.; Ma, J.X. Conjugated carbonyl compounds as high-performance cathode materials for rechargeable batteries. Chem. Mater. 2019, 31, 8582-8612. [CrossRef]

5. Lyu, H.L.; Sun, X.G.; Dai, S. Organic cathode materials for lithium-Ion batteries: Past, present, and future. Adv. Energy Sustain. Res. 2021, 2, 2000044. [CrossRef]

6. Lu, Y.; Zhang, Q.; Li, L.; Niu, Z.Q.; Chen, J. Design strategies toward enhancing the performance of organic electrode materials in metal-ion batteries. Chem 2018, 4, 2786-2813. [CrossRef]

7. Han, C.P.; Li, H.F.; Shi, R.Y.; Zhang, T.F.; Tong, J.; Li, J.Q.; Li, B.H. Organic quinones towards advanced electrochemical energy storage: Recent advances and challenges. J. Mater. Chem. A 2019, 7, 23378-23415. [CrossRef]

8. Yokoji, T.; Matsubara, H.; Satoh, M. Rechargeable organic lithium-ion batteries using electron-deficient benzoquinones as positive-electrode materials with high discharge voltages. J. Mater. Chem. A 2014, 2, 19347-19354. [CrossRef]

9. Liang, Y.L.; Zhang, P.; Chen, J. Function-oriented design of conjugated carbonyl compound electrodes for high energy lithium batteries. Chem. Sci. 2013, 4, 1330-1337. [CrossRef]

10. Liang, Y.L.; Zhang, P.; Yang, S.Q.; Tao, Z.L.; Chen, J. Fused heteroaromatic organic compounds for high-power electrodes of rechargeable lithium batteries. Adv. Energy Mater. 2013, 3, 600-605. [CrossRef]

11. Nagamura, N.; Taniki, R.; Kitada, Y.; Masuda, A.; Kobayashi, H.; Oka, N.; Honma, I. Electronic states of quinones for organic energy devices: The effect of molecular structure on electrochemical characteristics. ACS Appl. Energy Mater. 2018, 1, $3084-3092$. [CrossRef]

12. Cheng, L.; Assary, R.S.; Qu, X.H.; Jain, A.; Ong, S.P.; Rajput, N.N.; Persson, K.; Curtiss, L.A. Accelerating electrolyte discovery for energy storage with high-throughput screening. J. Phys. Chem. Lett. 2015, 6, 283-291. [CrossRef] [PubMed]

13. Zhang, Q.; Khetan, A.; Er, S. Comparison of computational chemistry methods for the discovery of quinone-based electroactive compounds for energy storage. Sci. Rep. 2020, 10, 22149. [CrossRef]

14. Zhang, Q.; Khetan, A.; Er, S. A quantitative evaluation of computational methods to accelerate the study of alloxazine-derived electroactive compounds for energy storage. Sci. Rep. 2021, 11, 4089. [CrossRef]

15. Tezsevin, I.; van de Sanden, M.C.M.; Er, S. High-throughput computational screening of cubic perovskites for solid oxide fuel cell cathodes. J. Phys. Chem. Lett. 2021, 12, 4160-4165. [CrossRef]

16. Sorkun, M.C.; Astruc, S.; Koelman, J.M.V.A.; Er, S. An artificial intelligence-aided virtual screening recipe for two-dimensional materials discovery. NPJ Comput. Mater. 2020, 6, 106. [CrossRef]

17. Sorkun, E.; Zhang, Q.; Khetan, A.; Sorkun, M.C.; Er, S. RedDB, A computational database of electroactive molecules for aqueous redox flow batteries. ChemRxiv 2021. [CrossRef]

18. Pineda Flores, S.D.; Martin-Noble, G.C.; Phillips, R.L.; Schrier, J. Bio-inspired electroactive organic molecules for aqueous redox flow batteries. 1. Thiophenoquinones. J. Phys. Chem. C 2015, 119, 21800-21809. [CrossRef]

19. Liu, C.F.; Neale, Z.G.; Cao, G.Z. Understanding electrochemical potentials of cathode materials in rechargeable batteries. Mater. Today 2016, 19, 109-123. [CrossRef]

20. Manzhos, S. Organic electrode materials for lithium and post-lithium batteries: An ab initio perspective on design. Curr. Opin. Green Sustain. Chem. 2019, 17, 8-14. [CrossRef]

21. Yokoji, T.; Kameyama, Y.; Maruyama, N.; Matsubara, H. High-capacity organic cathode active materials of 2,2'-bis-p-benzoquinone derivatives for rechargeable batteries. J. Mater. Chem. A 2016, 4, 5457-5466.

22. Hernández-Burgos, K.; Burkhardt, S.E.; Rodríguez-Calero, G.G.; Hennig, R.G.; Abruña, H.D. Theoretical studies of carbonyl-based organic molecules for energy storage applications: The heteroatom and substituent effect. J. Phys. Chem. C 2014, 118, 6046-6051. [CrossRef]

23. Kim, K.C.; Liu, T.Y.; Jung, K.H.; Lee, S.W.; Jang, S.S. Unveiled correlations between electron affinity and solvation in redox potential of quinone-based sodium-ion batteries. Energy Storage Mater. 2019, 19, 242-250. [CrossRef]

24. Botchkarev, A. Performance metrics (error measures) in machine learning regression, forecasting and prognostics: Properties and typology. arXiv 2018. arXiv:1809.03006.

25. Yao, M.; Senoh, H.; Yamazaki, S.I.; Siroma, Z.; Sakai, T.; Yasuda, K. High-capacity organic positive-electrode material based on a benzoquinone derivative for use in rechargeable lithium batteries. J. Power Sources 2010, 195, 8336-8340. [CrossRef] 
26. Shimizu, A.; Kuramoto, H.; Tsujii, Y.; Nokami, T.; Inatomi, Y.; Hojo, N.; Suzuki, H.; Yoshida, J.I. Introduction of two lithiooxycarbonyl groups enhances cyclability of lithium batteries with organic cathode materials. J. Power Sources 2014, 260, $211-217$. [CrossRef]

27. Kim, K.C.; Liu, T.Y.; Lee, S.W.; Jang, S.S. First-principles density functional theory modeling of Li binding: Thermodynamics and redox properties of quinone derivatives for lithium-ion batteries. J. Am. Chem. Soc. 2016, 138, 2374-2382. [CrossRef] [PubMed]

28. Barrès, A.L.; Geng, J.; Bonnard, G.; Renault, S.; Gottis, S.; Mentré, O.; Frayret, C.; Dolhem, F.; Poizot, P. High-potential reversible Li deintercalation in a substituted tetrahydroxy- $p$-benzoquinone dilithium salt: An experimental and theoretical study. Chem.- $A$ Eur. J. 2012, 18, 8800-8812. [CrossRef] [PubMed]

29. Wodrich, M.D.; Corminboeuf, C.; Schreiner, P.R.; Fokin, A.A.; Schleyer, P.V.R. How accurate are DFT treatments of organic energies? Org. Lett. 2007, 9, 1851-1854. [CrossRef]

30. Tang, M.; Zhu, S.L.; Liu, Z.T.; Jiang, C.; Wu, Y.C.; Li, H.Y.; Wang, B.; Wang, E.J.; Ma, J.; Wang, C.L. Tailoring $\pi$-conjugated systems: From $\pi-\pi$ stacking to high-rate-performance organic cathodes. Chem 2018, 4, 2600-2614. [CrossRef]

31. User Manual. Schrödinger: New York, NY, USA, 2019. Available online: https://www.schrodinger.com/ (accessed on 27 October 2021).

32. Roos, K.; Wu, C.J.; Damm, W.; Reboul, M.; Stevenson, J.M.; Lu, C.; Dahlgren, M.K.; Mondal, S.; Chen, W.; Wang, L.; et al. OPLS3e: Extending force field coverage for drug-like small molecules. J. Chem. Theory Comput. 2019, 15, 1863-1874. [CrossRef]

33. Niu, Z.H.; Wu, H.X.; Lu, Y.H.; Xiong, S.Y.; Zhu, X.; Zhao, Y.; Zhang, X.H. Orbital-dependent redox potential regulation of quinone derivatives for electrical energy storage. RSC Adv. 2019, 9, 5164-5173. [CrossRef]

34. Marten, B.; Kim, K.; Cortis, C.; Friesner, R.A.; Murphy, R.B.; Ringnalda, M.N.; Sitkoff, D.; Honig, B. New model for calculation of solvation free energies: Correction of self-consistent reaction field continuum dielectric theory for short-range hydrogen-bonding effects. J. Phys. Chem. 1996, 100, 11775-11788. [CrossRef]

35. Mohamadi, F.; Richards, N.G.J.; Guida, W.C.; Liskamp, R.; Lipton, M.; Caufield, C.; Chang, G.; Hendrickson, T.; Still, W.C. MacroModel-An integrated software system for modeling organic and bioorganic molecules using molecular mechanics. J. Comput. Chem. 1990, 11, 440-467. [CrossRef]

36. Stewart, J.J.P. MOPAC: A semiempirical molecular orbital program. J. Comput. Aided. Mol. Des. 1990, 4, 1-105. [CrossRef]

37. Bachrach, S.M. Jaguar 5.5. Schrödinger, Inc. J. Am. Chem. Soc. 2004, 126, 5018. [CrossRef]

38. te Velde, G.; Bickelhaupt, F.M.; Baerends, E.J.; Fonseca Guerra, C.; van Gisbergen, S.J.A.; Snijders, J.G.; Ziegler, T. Chemistry with ADF. J. Comput. Chem. 2001, 22, 931-967. [CrossRef]

39. Grimme, S.; Bannwarth, C.; Shushkov, P. A robust and accurate tight-binding quantum chemical method for structures, vibrational frequencies, and noncovalent interactions of large molecular systems parametrized for all spd-block elements $(\mathrm{Z}=1-86)$. J. Chem. Theory Comput. 2017, 13, 1989-2009. [CrossRef] [PubMed]

40. Elstner, M.; Porezag, D.; Jungnickel, G.; Elsner, J.; Haugk, M.; Frauenheim, T.; Suhai, S.; Seifert, G. Self-consistent-charge density-functional tight-binding method for simulations of complex materials properties. Phys. Rev. B 1998, 58, 7260-7268. [CrossRef]

41. Oliveira, A.F.; Philipsen, P.; Heine, T. DFTB parameters for the periodic table, part 2: Energies and energy gradients from hydrogen to calcium. J. Chem. Theory Comput. 2015, 11, 5209-5218. [CrossRef]

42. Hay, P.J.; Wadt, W.R. Ab initio effective core potentials for molecular calculations. Potentials for K to Au including the outermost core orbitale. J. Chem. Phys. 1985, 82, 299-310. [CrossRef] 